\title{
Singular enrichment functions for Helmholtz scattering at corner locations using the Boundary Element Method
}

\author{
B. Gilvey ${ }^{* \dagger}$, J. Trevelyan ${ }^{1}$, G. Hattori ${ }^{2}$ \\ ${ }^{1}$ Department of Engineering, Durham University, South Road, Durham DH1 3LE, UK \\ ${ }^{2}$ Department of Engineering, University of Cambridge, Trumpington Street, Cambridge, UK
}

\begin{abstract}
SUMMARY
In this paper we use an enriched approximation space for the efficient and accurate solution of the Helmholtz equation in order to solve problems of wave scattering by polygonal obstacles. This is implemented in both Boundary Element Method (BEM) and Partition of Unity Boundary Element Method (PUBEM) settings. The enrichment draws upon the asymptotic singular behaviour of scattered fields at sharp corners, leading to a choice of fractional order Bessel functions that complement the existing Lagrangian (BEM) or plane wave (PUBEM) approximation spaces. Numerical examples consider configurations of square scattering objects, subject to the Neumann 'sound hard' boundary conditions, demonstrating that the approach is a suitable choice for both convex scatterers and also for multiple scattering objects that give rise to multiple reflections. Substantial improvements are observed, significantly reducing the number of degrees of freedom required to achieve a prescribed accuracy in the vicinity of a sharp corner.

Copyright (c) 2017 John Wiley \& Sons, Ltd.
\end{abstract}

Received ...

KEY WORDS: BEM, PUBEM, Bessel functions

\section{INTRODUCTION}

Acoustic wave propagation is a broad field of study, in which the Finite Element Method (FEM) 10 and Boundary Element Method (BEM) are popular approaches for numerical simulation. It is well known that as frequency $\omega$ increases, and the wavelength $\lambda$ decreases relative to the domain of interest, the computational expense using these methods may become prohibitively large. The design of numerical methods to ameliorate the computational complexity is an active field of research.

While we recognise alternative approaches to accelerate computations in high-frequency acoustics, based on fast multipole expansions [1], low-rank matrix approximations [2] and use of optimised linear algebra routines [3], in this article we focus on the class of methods that reduce the size of the required linear system by utilising enrichment, which can be viewed as the use of operator-specific basis functions. One way to achieve this is to use a high-order basis, for example in the Isogeometric Finite Element Method (IGAFEM) [4], and with Bernstein-Bézier polynomials [5] which admit acceleration using static condensation. Other approaches involving oscillatory basis functions have their roots in Trefftz methods, in which functions that are known local solutions to the Partial Differential Equation (PDE) at hand are inserted into the approximation space. A relevant comparison with high order schemes is made in [6]. Methods employing a wave basis draw upon the Partition of Unity Method [7], and these include the Partition of Unity Finite Element

\footnotetext{
*Correspondence to: B. Gilvey, Department of Engineering, Durham University, South Road, Durham DH1 3LE, UK.

${ }^{\dagger}$ E-mail: benjamin.gilvey@durham.ac.uk 
Method (PUFEM) [8, 9], the Discontinuous Enrichment Method [10], the Variational Theory of Complex Rays (VTCR) [11], the Ultraweak Variational Formulation (UWVF) [12] and the Plane Wave Discontinuous Galerkin Method (PWDG) [13, 14].

The above methods involve volumetric discretisation. When considering problems of acoustic radiation and/or scattering in infinite domains, however, it is preferable to employ methods which require information only on the boundary of the scattering object, and the BEM has become a standard approach. A clear benefit is a reduction in dimensionality of the problem, but another advantage is that the Sommerfeld radiation condition [15] is satisfied by construction. Thus there is no requirement to truncate the domain and impose artificial non-reflecting boundary conditions. In order to address the computational complexity at high frequencies, a BEM formulation based on the

35 Partition of Unity Method (PUBEM) was developed by Perrey-Debain et al. [16-18]. In these works, the use of a plane wave basis was shown to reduce the required number of degrees of freedom (DoF) per wavelength (a parameter we denote $\tau$ ) from between 8-10 when using conventional elements to approximately 2.5. A highly efficient wave basis approach tailored for scattering by a single convex polygonal scatterer was developed by Chandler-Wilde [19], and was further developed into the 40 Hybrid Numerical Asymptotic approach [20].

While PUBEM provides a considerable improvement on BEM for smooth scattering objects, its advantages over BEM when considering polygonal scattering objects become less substantial. This results from the plane wave basis not efficiently resolving the singular behaviour around sharp corners. There have been attempts to mitigate the expense resulting from corner singularities using

45 a graded mesh along with a preconditioning strategy [21] and utilising a fast solver [22]. Alternative approaches rely on injecting the correct asymptotic local behaviour at corner locations into the approximation space. This can been seen for Laplace operators in [23], external problems using the UWVF [24], and successfully combined with the Method of Fundamental Solutions (MFS) [25,26]. More recently, the impact that the singularity has on pollution in FEM for large $k$ is shown in [27]. The extension of this enrichment strategy to BEM and PUBEM is the focus of this paper.

The paper is organised in the following way. In section 2 we present the relevant Boundary Integral Equation (BIE) and its discrete solution using the BEM formulation. In section 3 we introduce the corner enrichment strategy, and test the scheme using a selection of numerical examples in section 4 . We close with some concluding remarks in section 5.

\section{BOUNDARY INTEGRAL EQUATION FORMULATION}

55 In this section the BIE is formulated, and the fundamental contribution highlighted from the enrichment functions employed. Consider a sound-hard polygonal object $\Omega_{s} \subset \mathbb{R}^{2}$ having boundary $\Gamma$, and the acoustic wave propagation in the infinite, fluid-filled region $\Omega_{f}=\mathbb{R}^{2} \backslash \Omega_{s}$ in the exterior when the scatterer is impinged by an incident plane wave. The problem is outlined by beginning with the time-harmonic reduction of the wave equation, the Helmholtz equation

$$
\nabla^{2} u(\mathbf{x})+k^{2} u(\mathbf{x})=0, \quad \mathbf{x} \in \Omega_{f}
$$

60 where the wavenumber $k=2 \pi / \lambda$. The unknown potential is $u \in \mathbb{C}$ and $\nabla^{2}$ is the Laplacian operator. The sound-hard scatterer is imposed through a Neumann boundary condition

$$
\nabla u(\mathbf{x}) \cdot \mathbf{n}=0, \quad \mathbf{x} \in \Gamma
$$

Making use of Green's second identity the Helmholtz equation can be expressed in an equivalent form of a BIE [28], i.e.

$$
c(p) u(p)+\int_{\Gamma} \frac{\partial G(p, q)}{\partial \mathbf{n}} u(q) d \Gamma_{q}=\int_{\Gamma} \frac{\partial u(q)}{\partial \mathbf{n}} G(p, q) d \Gamma_{q}+u^{i n c}(p), \quad p \in \Gamma
$$

where the first term arises from the presence of the strongly singular integral on the left hand side,

65 with $c(p)=\frac{1}{2}$ on a smooth boundary $\Gamma$, but varying as a function of the exterior angle at nonsmooth boundary points. The points $p=\left(x_{p}, y_{p}\right)$ and $q=\left(x_{q}, y_{q}\right)$ are the so called 'source' and 
'field' points respectively. $u^{i n c}$ is the incident wave and the vector $\mathbf{n}$ is the unit normal vector outward pointing from $\Omega_{f}$. As the focus of this paper remains in two-dimensional space, the Green's function $G(p, q)$ is

$$
G(p, q)=\frac{i}{4} H_{0}^{(1)}(k r),
$$

70 where $H_{0}^{(1)}$ is the Hankel function of the first kind and of order zero, and $r$ is the Euclidian distance between $p$ and $q$. The acoustically rigid Neumann boundary condition (2) causes the third term of (3) to vanish. Solution of (1) by the BEM is achieved by discretising the BIE (3) and making some assumption about the form of $u$ over each element of the discretisation. Conventionally one might use low order polynomial shape functions to represent the varying acoustic potential over an element 75 e, i.e.

$$
u=\sum_{j=1}^{J} N_{j}(\xi) u_{j}^{e}
$$

in which $N_{j}$ denotes the polynomial shape function (typically Lagrangian) for node $j, \xi \in[-1,1]$ is the usual parametric variable describing the element, $u_{j}^{e}$ are the unknown nodal potential values at node $j$ on element $e$, and $J$ is the number of nodes in the element. This results in the discretised form of (3) in a BEM setting as follows

$$
c(p) u(p)+\sum_{e=1}^{E} \sum_{j=1}^{J} \int_{-1}^{1} \frac{\partial G(p, q)}{\partial \mathbf{n}} N_{j}(\xi) J^{e} d \xi u_{j}^{e}=u^{i n c}(p),
$$

where $E$ is the total number of elements and $J^{e}$ is the Jacobian of the geometric mapping $(x, y) \rightarrow \xi$. In PUBEM the polynomial basis is enriched with plane waves, such that the unknown potential is written as a linear combination of plane waves propagating in different directions, i.e.

$$
\begin{gathered}
u=\sum_{j=1}^{J} \sum_{m=1}^{M} N_{j}(\xi) A_{j m} \mathrm{e}^{i k \mathbf{d}_{j m} \cdot \mathbf{q}}, \\
\mathbf{d}_{j m}=\left(\cos \theta_{j m}, \sin \theta_{j m}\right), \quad \theta_{j m}=\frac{2 \pi(m-1)}{M}
\end{gathered}
$$

where $A_{j m}$ are the unknown plane wave amplitudes, $\mathbf{d}_{j m}$ are the direction vectors of the plane waves, $i=\sqrt{-1}$ and $M$ is the number of plane waves considered per node. Therefore the discretised form of (3) in a PUBEM setting becomes

$$
c(p) u(p)+\sum_{e=1}^{E} \sum_{j=1}^{J} \sum_{m=1}^{M} \int_{-1}^{1} \frac{\partial G(p, q)}{\partial \mathbf{n}} N_{j}(\xi) \mathrm{e}^{i k \mathbf{d}_{j m} \cdot \mathbf{q}} J^{e} d \xi A_{j m}^{e}=u^{i n c}(p) .
$$

While this can be considered in a Galerkin procedure, in the current work the collocation form of BEM is employed, so that (9) is collocated at a sufficient number of points $p$ to yield a solvable linear system. It should be noted that with the plane wave expansion (7) the number of degrees of 90 freedom greatly exceeds the number of nodes, requiring collocation at many non-nodal locations. Further, it is well known that solutions of (3) become non-unique at frequencies corresponding to the eigenfrequencies of the associated interior problem formed on the same boundary $\Gamma$. In the interest of simplicity, the Combined Helmholtz Integral Equation Formulation (CHIEF) [29] is employed here to overcome the system degeneracy. CHIEF requires additional collocation points located in 95 the interior domain $\Omega_{s}$ of the scattering object, resulting in an overdetermined system of equations, that requires an appropriate solver. It should be noted that there is a popular alternative method of Burton and Miller [30] which can be regularised as in [31,32] reducing the hypersingular integrals to weakly singular.

It is commonly observed that enriched formulations suffer from ill-conditioning of the resulting linear system. However, we note that the ill-conditioning in PUBEM can be managed by (i) ensuring 
that the problem is suitably discretised by controlling $\tau$ to be not too large, (ii) oversampling with use of more collocation points than strictly necessary, and (iii) for the more severely ill-conditioned systems, using a truncated Singular Value Decomposition (SVD) to solve the overdefined system of equations.

\section{MODIFIED BASIS FUNCTIONS}

In this section both Extended Boundary Element Method (XBEM) and Partition of Unity Extended Boundary Element Method (PUXBEM) bases are proposed. To develop our XBEM formulation, we augment the polynomial expansion of potential by including additional functions, denoted $\psi$, representing the singular behaviour at corners, so that over element $e$ (5) becomes replaced by

$$
u=\sum_{j=1}^{J} N_{j}(\xi) u_{j}^{e}+\sum_{l=1}^{L} \psi_{l}(\xi) \bar{u}_{l}^{e}
$$

Thus we augment the discretised BIE (6) to formulate XBEM, as follows

$$
c(p) u(p)+\sum_{e=1}^{E} \sum_{j=1}^{J} \int_{-1}^{1} \frac{\partial G(p, q)}{\partial \mathbf{n}} N_{j}(\xi) J^{e} d \xi u_{j}^{e}+\sum_{e=1}^{E} \sum_{l=1}^{L} \int_{-1}^{1} \frac{\partial G(p, q)}{\partial \mathbf{n}} \psi_{l}(\xi) J^{e} d \xi \bar{u}_{l}^{e}=u^{i n c}(p)
$$

110 The coefficients $\bar{u}_{l}^{e}$ therefore become part of the solution vector alongside the other unknowns $u_{j}^{e}$. Once the BEM system has been solved, these become available and the potential at any location on $\Gamma$ can be recovered through (10). The corner enrichment can be extended to the PUBEM technique (9) to provide the new PUXBEM formulation. Here, we augment the plane wave expansion of potential by including the additional functions $\psi$, so that over element $e$ (7) becomes replaced by

$$
u=\sum_{j=1}^{J} \sum_{m=1}^{M} N_{j}(\xi) A_{j m}^{e} \mathrm{e}^{i k \mathbf{d}_{j m} \cdot \mathbf{q}}+\sum_{l=1}^{L} \psi_{l}(\xi) B_{l}^{e},
$$

and the corresponding discretised BIE becomes

$c(p) u(p)+\sum_{e=1}^{E} \sum_{j=1}^{J} \sum_{m=1}^{M} \int_{-1}^{1} \frac{\partial G(p, q)}{\partial \mathbf{n}} N_{j}(\xi) \mathrm{e}^{i k \mathbf{d}_{j m} \cdot \mathbf{q}} J^{e} d \xi A_{j m}^{e}+\sum_{e=1}^{E} \sum_{l=1}^{L} \int_{-1}^{1} \frac{\partial G(p, q)}{\partial \mathbf{n}} \psi_{l}(\xi) J^{e} d \xi B_{l}^{e}=u^{i n c}(p)$.

Once again, the coefficients $B_{l}^{e}$ are auxiliary unknowns to be solved for in the BEM system.

The choice of the form of enrichment functions $\psi_{l}$ is informed by the asymptotic potential behaviour around a corner. From [33] the asymptotic behaviour locally to the corner (for the soundhard Neumann case) can be shown to be

$$
u\left(r_{b}, \theta_{b}\right) \approx \sum_{n=1}^{N} J_{n \alpha}\left(k r_{b}\right) \cos n \alpha \theta_{b}, \quad 0<\frac{\pi}{\alpha}<2 \pi,
$$

where $\alpha$ is such that the exterior angle at the corner is $\pi / \alpha, r_{b}$ is the distance from the corner and angle $\theta_{b}$ is measured from one of the planar surfaces at the corner as shown in Fig. 1. In Fig. 2 we plot the family of Bessel functions $J_{n \alpha}\left(k r_{b}\right)$ for the case $k=20$ and taking $\alpha=2 / 3$ to indicate a $90^{\circ}$ corner. The singularity in the gradient of the Bessel function $J_{2 / 3}\left(k r_{b}\right)$ is evident at $r_{b}=0$, and it is this form that is expected to provide a suitable local enrichment in the vicinity of the corner. Further, since the Bessel functions considering $n>1$ do not exhibit a singular gradient, we suppose that these will provide only a negligible improvement in the approximation over the piecewise polynomial (BEM) or plane wave (PUBEM) basis functions. Therefore, unlike Luostari et al. [24] and Barnett \& Betcke [25], we proceed taking $N=1$, i.e. taking only the first Bessel 
term in the series (14). Further, we note that the behaviour of this Bessel function for small $r_{b}$ is of the form $r_{b}^{\alpha}$. This latter function will be faster to compute than the full Bessel function yet will still contain the important asymptotic behaviour. For this reason we investigate the use of two candidate forms, $\psi_{1, l}$ and $\psi_{2, l}$, of the enrichment function, being

$$
\begin{gathered}
\psi_{1, l}=r_{b}^{\alpha} \cos l \alpha \theta_{b} \\
\psi_{2, l}=J_{l \alpha}\left(k r_{b}\right) \cos l \alpha \theta_{b} .
\end{gathered}
$$

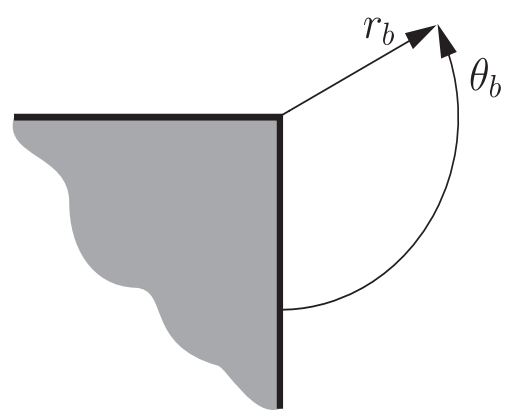

Figure 1. Polar coordinate system local to a corner.

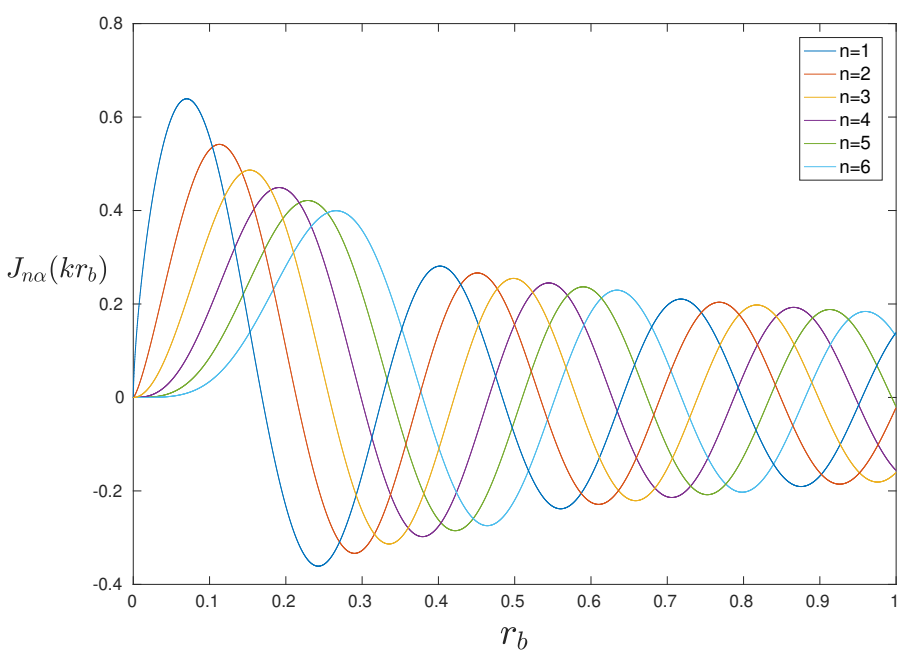

Figure 2. Bessel functions with $n=1-6, \alpha=\frac{2}{3}$ and $k=20$.

The cosine term in (14)-(16) describes the smooth variation in potential in the circumferential direction in the vicinity of the corner. While this is of great importance in finite element and UWVF implementations, we note that in a BEM context we are required to evaluate only the trace of the functions on the boundary, and for the flat-sided polygonal geometries considered in this paper we have constant $\theta_{b}$ over each edge. The two edges meeting at any right-angled corner, for example, would have constant $\theta_{b}=0$ and $\theta_{b}=3 \pi / 2$. This admits the possibility of simplifying the enrichment functions by omitting the trigonometric term, leaving $\psi_{1, l}=r_{b}^{\alpha}, \psi_{2, l}=J_{l \alpha}\left(k r_{b}\right)$. This discussion also suggests that there are two options for the Bessel based enrichment. Firstly, to use two DoF per Bessel function, i.e. a separate DoF corresponding to each of the edges meeting at the corner in question. We refer to this as formulation $A$. Secondly, to include each Bessel function as a single DoF, inserting $\theta_{b}$ into the argument, which will take the value of either 0 or $3 \pi / 2$, for a right-angled corner. This will be referred to as formulation $B$. The distinction is highlighted below. 
Formulation $A$ :

$$
\begin{aligned}
& \bar{u}_{L}^{e} \neq \bar{u}_{1}^{e+1} \\
& B_{L}^{e} \neq B_{1}^{e+1}
\end{aligned}
$$

Formulation $B$ :

$$
\begin{aligned}
& \bar{u}_{L}^{e}=\bar{u}_{1}^{e+1} \\
& B_{L}^{e}=B_{1}^{e+1}
\end{aligned}
$$

We note that by including the Bessel function or plane wave enrichment, oscillatory integrals are introduced. At present the integration scheme employed is a sub-divided Gauss-Legendre scheme. Since the focus of the current work is the numerical behaviour of BEM schemes with corner enrichment, we use a larger number of Gauss points than is necessary in order to eliminate as far as possible any errors due to integration.

\section{NUMERICAL TESTING}

This section first considers scattering by a single square in an infinite domain, such as the case illustrated in Fig. 3 (the case $k=50$ is shown). Both the number of degrees of freedom (DoF) used and the radius $r_{i p}$ at which the evaluation points are located are varied, as defined in Fig. 4. We consider an $L_{2}$ relative error $\epsilon$, taken over this circular contour, and defined as

$$
\epsilon=\frac{\left\|u-u_{r e f}\right\|_{L_{2}}}{\left\|u_{r e f}\right\|_{L_{2}}}
$$

where $u^{r e f}$ is a reference solution. The analysis considers the influence of wavenumber $k$ on the error norm, and a further example of scattering by two square objects is investigated to demonstrate the efficacy of XBEM and PUXBEM in the presence of reflections. With the exception of section 4.1.4, formulation $A$ is employed for the Bessel based enrichment.
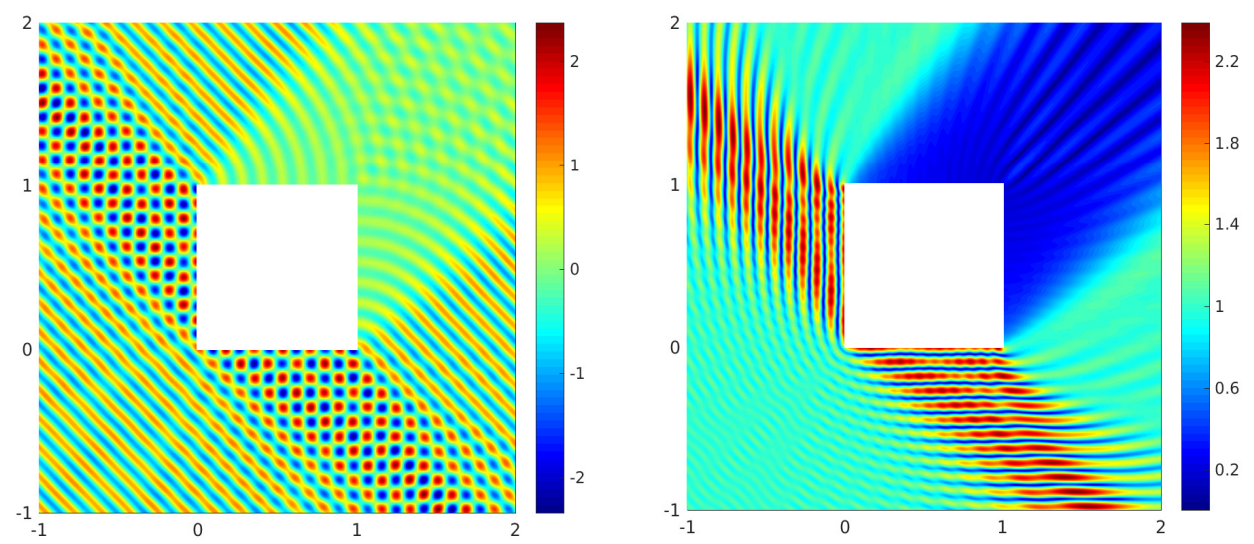

Figure 3. (left) Real part of the total potential, (right) absolute value of total potential. Unit square scatterer for the case $k=50$. 


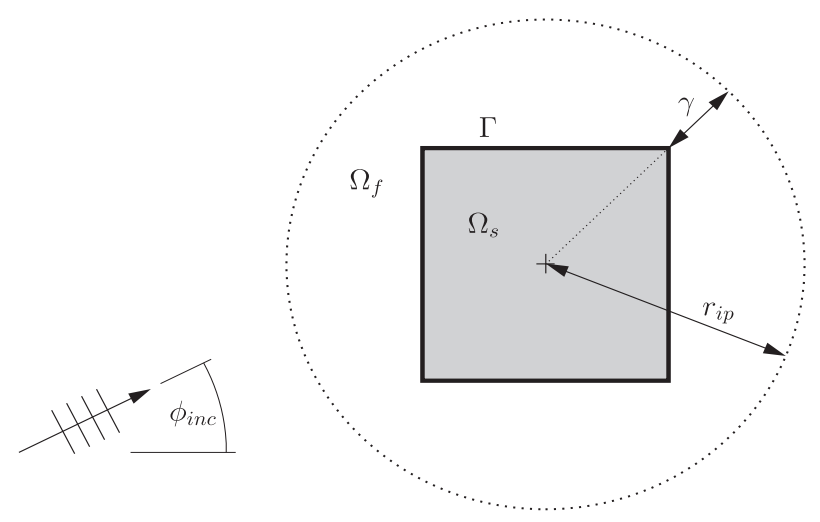

Figure 4. Internal point locations for a square scatterer.

\subsection{Square scattering object}

Consider a unit square being impinged by a plane wave $u^{i n c}$ propagating in direction $\phi_{i n c}=\frac{\pi}{4}$. In our first analysis we take the wavenumber $k=20$ and the geometry dictates that $\alpha=\frac{2}{3}$. We evaluate the potential over a ring of points at $r_{i p}=\sqrt{0.5^{2}+0.5^{2}}+\gamma$, and plot the relative error $\epsilon$ for the case $\gamma=1$ in Fig. 5.

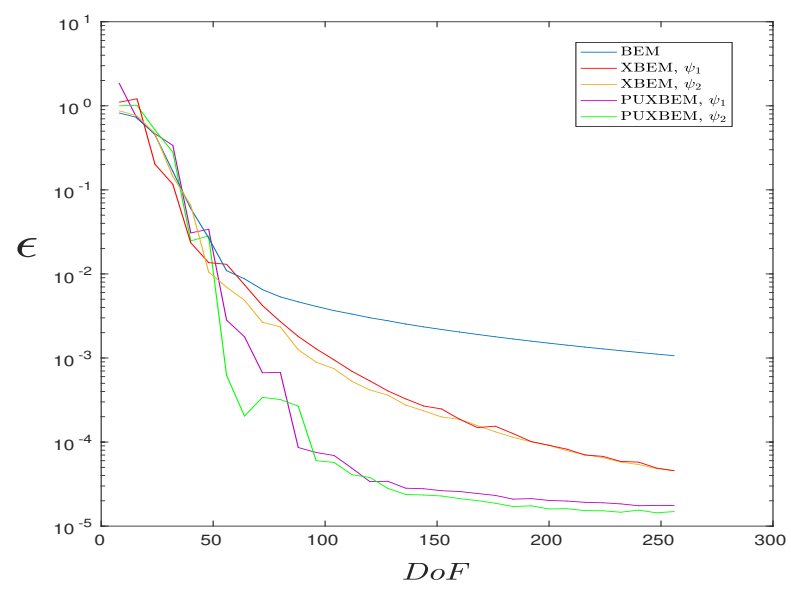

Figure 5. Relative error vs. DoF.

It is evident that a notable improvement is seen when the corner enrichment is included, with the PUXBEM formulation outperforming the XBEM. The enrichment function forms $\psi_{1, l}$ and $\psi_{2, l}$ offer similar benefit, with slightly more favourable results found using $\psi_{2, l}$.

Extending the analysis of the unit square by varying $k$ provides further insight. This is shown by a comparison made in Fig. 6 for $k$ in the range 1 to 40, evaluated using each respective method with the number of DoF fixed at 128. The results confirm that XBEM and PUXBEM outperform BEM considerably. It is worth noting that as $k$ increases, so does the XBEM error, eventually approaching the BEM result. This is due to the fact that the $128 \mathrm{DoF}$ model becomes less able to resolve the solution generally over the square with the (predominantly) polynomial basis functions in XBEM. Conversely with PUXBEM, as $k$ increases, the plane wave basis is able to maintain accuracy over a larger frequency range.

Further comparison of the unit square case is made in Table I, which shows the errors for varying

$180 \gamma$ obtained using a fixed DoF taking $k=20$ throughout. The table highlights the fact that overall accuracy increases as we consider results at points further from the scatterer. It is interesting 


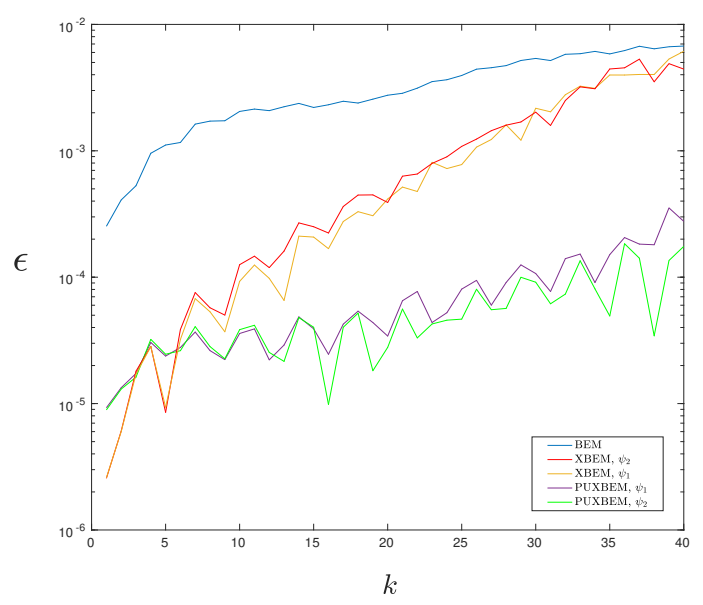

Figure 6. Relative error vs. $k$ using 128 DoF.

to observe that it is not only locally around the corners that the additional enrichment increases accuracy; significant reduction in error is also seen in the far field.

\begin{tabular}{|c|c|c|c|}
\hline$\gamma$ & BEM & XBEM & PUXBEM \\
\hline 1 & $2.8354 e-03$ & $4.3427 e-04$ & $2.7960 e-05$ \\
\hline 2 & $2.2018 e-03$ & $3.4139 e-04$ & $2.1726 e-05$ \\
\hline 3 & $1.8703 e-03$ & $2.9102 e-04$ & $1.8458 e-05$ \\
\hline 4 & $1.6527 e-03$ & $2.5755 e-04$ & $1.6312 e-05$ \\
\hline 5 & $1.4947 e-03$ & $2.3311 e-04$ & $1.4753 e-05$ \\
\hline \multicolumn{4}{|c}{ Table I. Relative error $\epsilon$ using 128 DoF for $\gamma=1-5}$.
\end{tabular}

4.1.1. Use of blending functions It is clear that the proposed corner enrichment functions $\psi_{1, l}$ and $\psi_{2, l}$ will (apart from a few special cases at the roots of the Bessel function) be non-zero for $r_{b}>0$. So, considering an enrichment function over an edge of the scatterer described by $0<r_{b}<L$, with $r_{b}=0$ at the corner we are enriching, use of these enrichment functions will then not enforce continuity of the potential at $r_{b}=L$. Restricting our analysis now to the formulation found most favourable in the previous section, i.e. PUXBEM with $\psi_{2, l}$, we focus on modifying this case to observe the impact of the discontinuity. In this study, we multiply $\psi_{2, l}$ by a tapering Hermite function that decays to zero at $r_{b}=L$. In this fashion $\psi_{2, l}$ is redefined as

$$
\psi_{2, l}=J_{l \alpha}\left(k r_{b}\right) \cos \left(l \alpha \theta_{b}\right) w(\eta)
$$

where

$$
\begin{gathered}
\eta=\frac{2 r_{b}}{L-\beta}-\frac{L+\beta}{L-\beta} \\
w(\eta)= \begin{cases}1, & \text { if } r_{b}<\beta \\
0.25(1-\eta)^{2}(2+\eta), & \text { otherwise }\end{cases}
\end{gathered}
$$

The resulting function is shown in Fig. 7. The tapering can begin at any desired point in the interval, depending on the choice of parameter $\beta$. The results displayed in Fig. 8 and Fig. 9 show that blending actually has a detrimental effect on the solution, presumably because the enrichment function is no longer the same as the theoretical asymptotic behaviour for $r_{b}>\beta$. Not only are the results less accurate but they are also less stable than those using unblended corner enrichment functions. 

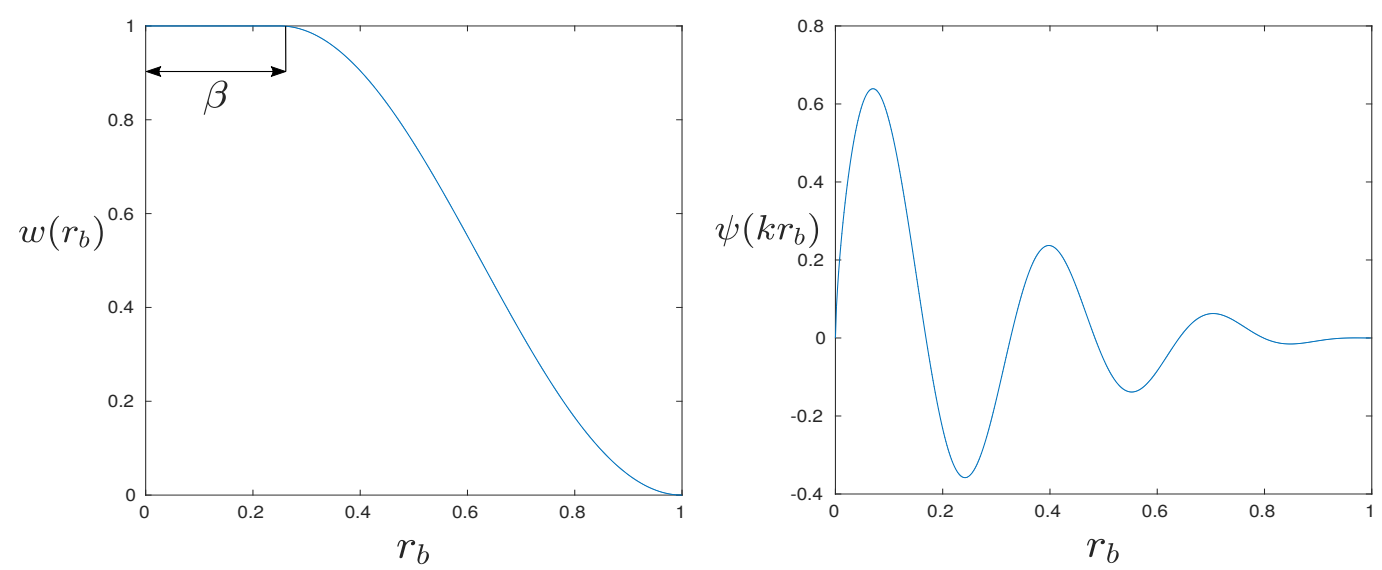

Figure 7. (left) Hermite blending function, (right) Blended Bessel function with $n=1, k=20$ and $\alpha=\frac{2}{3}$.

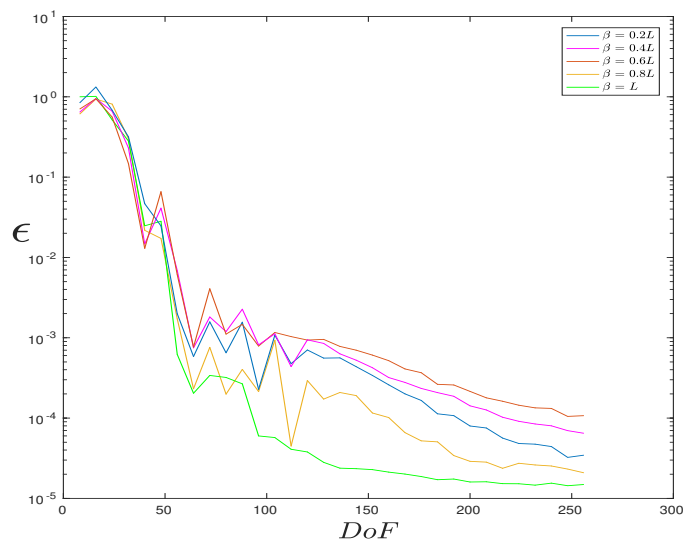

Figure 8. Relative error vs. DoF for $\gamma=1$, varying $\beta$ where $L$ is the element length.

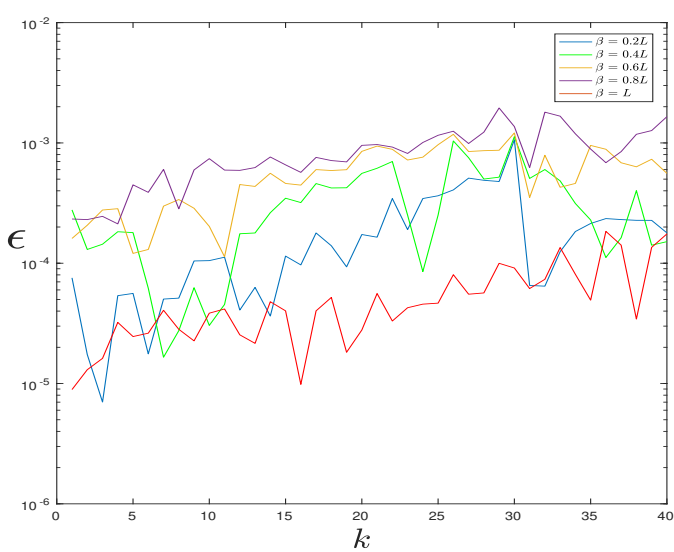

Figure 9. Relative error vs. $k$ using $128 \mathrm{DoF}$, varying $\beta$ where $L$ is the element length.

We have tested five different values of $\beta=(0.2 L, 0.4 L, 0.6 L, 0.8 L, L)$ in producing these results, and one may note that use of $\beta=L$ is consistent with no blending function having been applied. One 
interesting observation from these figures is that if we rank the values of $\beta$ in order of their general performance we find a sequence $\beta=L$ (best), $0.2 L, 0.4 L, 0.6 L, 0.8 L$ (worst). The conclusion is that it is best to remove the blending function entirely (accepting a small discontinuity at $r=L$ ) but, if one is going to include blending then it is best to use a small $\beta$. Our interpretation of this rather counter-intuitive behaviour is that a large $\beta$ will give rise to a steep blending function that means the blended enrichment function $\psi_{2, l}$ is less faithful to the theoretical asymptotic behaviour in (14) in the region $\beta<r_{b}<L$.

4.1.2. Accuracy locally at a corner The analysis is now taken closer to the corner locations by distributing internal points uniformly on four arcs according to Fig. 10, each of radius $\gamma$ centered around a corner. This defines a composite contour

$$
\Gamma_{R}=\bigcup_{i=1}^{i=4} \Gamma_{R}^{i} .
$$

The error $\epsilon$ is shown in Fig. 11 over a range of $\gamma=0.01-0.2$. The number of degrees of freedom is fixed at 128 , giving $\tau \approx 10$, which is a discretisation that would normally be sufficient for unenriched BEM schemes. In the presence of corners, it is clear that the unenriched BEM is not capable of achieving engineering accuracy (for which we take a common definition of $1 \%$ error) locally around the corners at this discretisation.

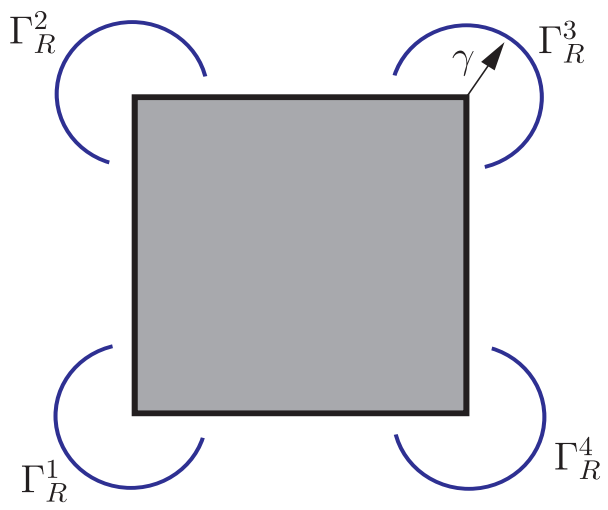

Figure 10. Internal point locations for single square scattering object.

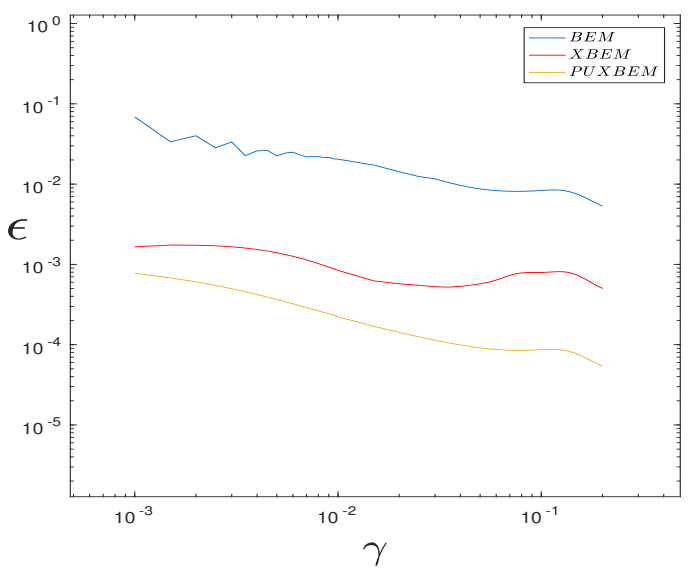

Figure 11. Relative error vs. $\gamma$ using 128 DoF. 
4.1.3. Conditioning It is commonly reported that enriched numerical schemes, particularly plane wave enriched schemes, suffer from a degree of ill conditioning. This is true of the current scheme, as illustrated in Fig. 12 which shows the condition number $\kappa$ of the system matrix generated for the three different numerical methods. The case $k=20$ for the square scatterer is shown. It can clearly be seen that the addition of eight degrees of freedom, turning the BEM scheme into XBEM, causes approximately two orders of magnitude increase in $\kappa$, though not to an extent that would interfere with the ability of solvers to reach an accurate solution. The significantly worse conditioning of PUXBEM derives from the plane wave basis and is consistent with the literature in these methods. It has already been seen in Fig. 11 that PUXBEM provides the highest accuracy of the three schemes, but the ill conditioned system requires an appropriate solver to be employed. In the current work we use the MATLAB backslash operator.

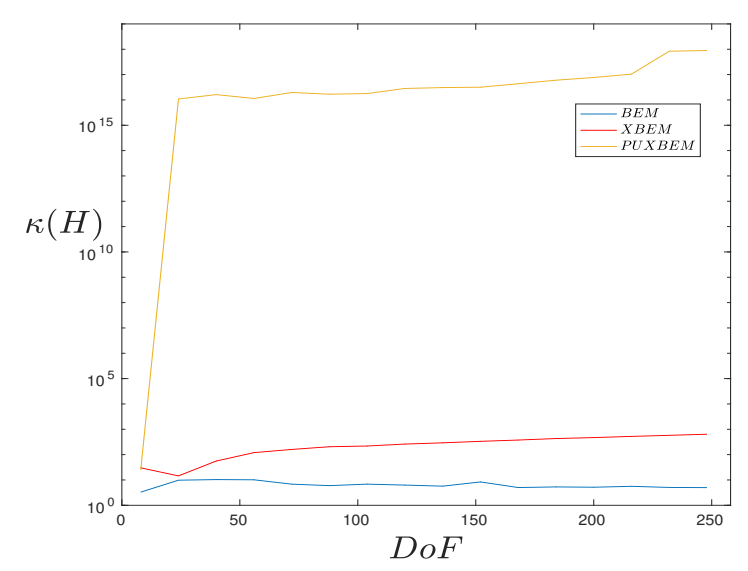

Figure 12. Condition number $\kappa$ of the $H$ matrix vs. degrees of freedom.

We note that Antunes [34] has recently presented a revised set of oscillatory basis functions for the Method of Fundamental Solutions that eliminates the problem of the ill-conditioning inherent in the use of a plane wave basis. However, if this approach were applied to PUBEM or PUXBEM, the increased computational demands required for basis function evaluation would be too high a price to pay for the improved conditioning when, as we have demonstrated, PUBEM and PUXBEM are very capable of producing highly accurate solutions in spite of the poor conditioning of the linear system.

4.1.4. Single degree of freedom per Bessel function In all of the examples shown up until this point, formulation $A$ is employed. In this section formulation $B$ is used to provide a comparison. We recall that in the formulation $A$, there are two degrees of freedom for the Bessel enrichment at a corner, while in formulation $B$ these are combined into a single degree of freedom at each corner as suggested by the trigonometric variation in (14). In Fig. 13 formulation $A$ and formulation $B$ are compared. While formulation $B$ results offer an improvement on traditional BEM and are slightly smoother, at no point do they outperform the original test cases, using formulation $A$. For this reason we proceed with formulation $A$ only.

While readers might question why the idea for formulation $B$ has been presented in this paper only to be found inferior, we emphasise that it has been included because it is the more faithful of the two formulations in representing the leading order term in the series (14) giving the theoretical asymptotic behaviour. It is an interesting result to see this outperformed consistently by the formulation $A$ which ignores the $\cos l \alpha \theta_{b}$ variation in the circumferential direction. 


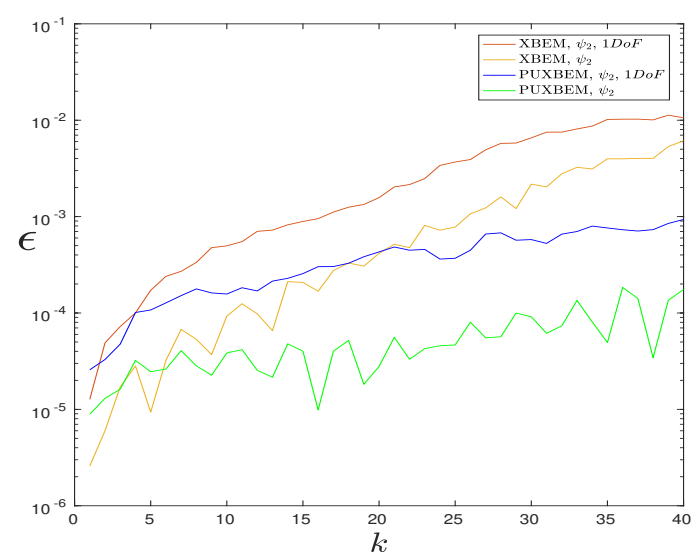

Figure 13. Relative error vs. DoF using a single DoF per $\psi_{2}$, marked $1 D o F$ and using a DoF for each edge. The single DoF results were obtained using $124 \mathrm{DoF}$ and the two DoF results used 128 DoF.

\subsection{Multiple square scattering}

One of the benefits of using a basis comprising multiple plane waves is that it provides an attractive method not only for single, convex scatterers, but also without modification for nonconvex scatterers, multiple scatterers, or other cases in which multiple internal reflections occur. For this reason in our final example we consider the scattering of an incident wave by a structure containing two squares. The problem is illustrated in Fig. 14, showing the geometry of the two unit squares along with their total potential field in which reflections between the scatterers can clearly be seen. As before we consider an incident plane wave propagating in an angle of $\pi / 4$ from the lower left corner.
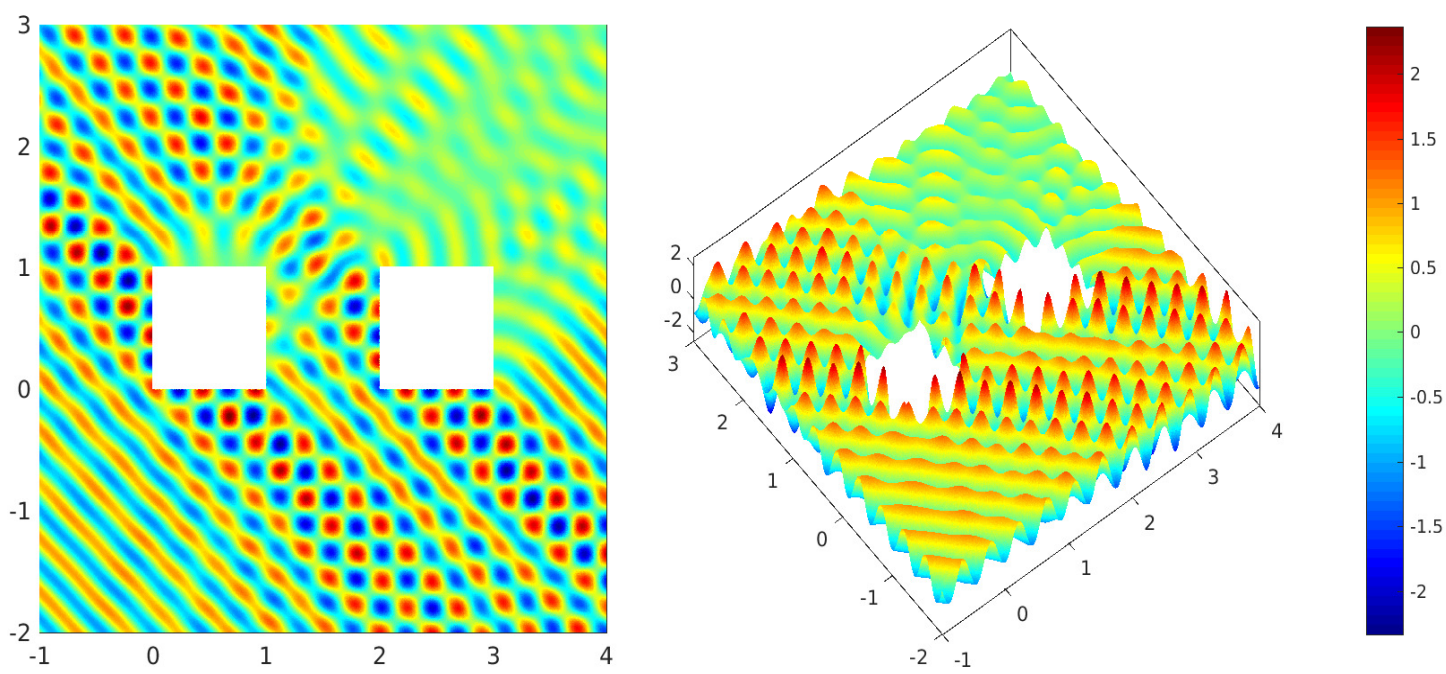

Figure 14. Real part of total potential field resulting from a configuration of two squares being impinged by an $\phi_{\text {inc }}=\frac{\pi}{4}$.

Numerical testing shows that the benefits enjoyed by the Bessel function enrichment for the single scattering object case directly translate to the multiple scattering arrangement. For this case, we find 
it useful to define a different metric. We first distribute internal points as shown in Fig. 10 (but for eight arcs), each of radius $\gamma$ centered around a corner. Defining a composite contour $\Gamma_{R}$, for the

evaluation of results, as

$$
\Gamma_{R}=\bigcup_{i=1}^{i=8} \Gamma_{R}^{i}
$$

we consider the following norm, $Q$, of the solution as

$$
Q=\|u\|_{L_{2}\left(\Gamma_{R}\right)} .
$$

A sufficiently small $\gamma=0.01$ is selected to produce the results in Fig. 15. The impact of the singularity is clearly visible as both XBEM and PUXBEM converge to the same result almost immediately, whereas a conventional unenriched BEM scheme oscillates gradually towards the same result.

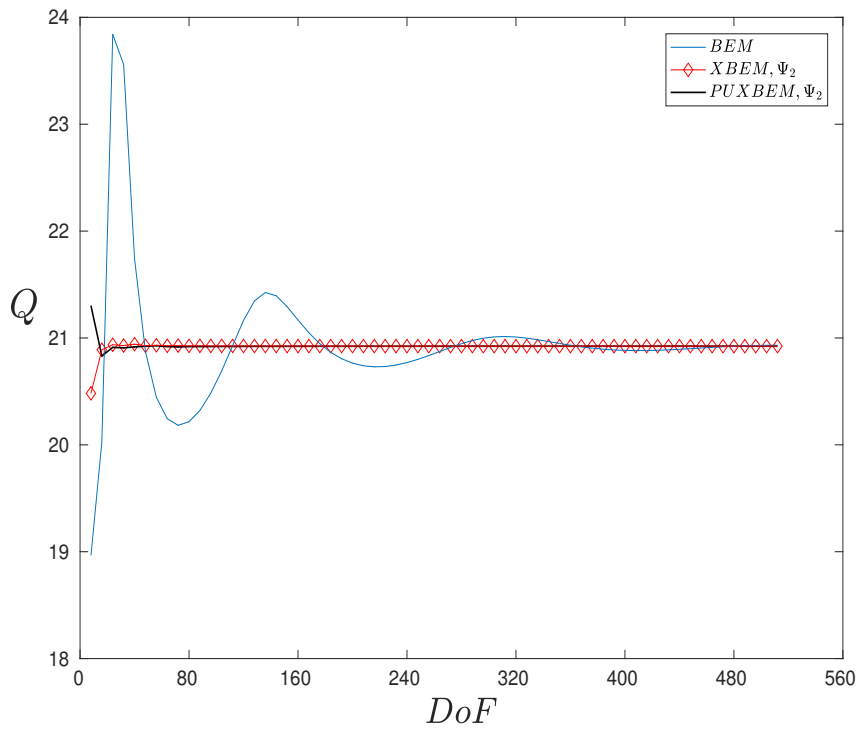

Figure 15. $L_{2}$ norm vs. DoF at internal points around two square scattering objects, where $\gamma=0.01$.

\subsection{Triangular scattering object}

Finally, we consider a unit triangle being impinged by a plane wave $u^{i n c}$ propagating in the direction $\phi_{i n c}=\frac{3 \pi}{2}$. In this example, the geometry dictates that $\alpha=\frac{3}{5}$. It is evident from Fig. 6 that XBEM is optimal for low $k$ and PUXEM optimal for larger $k$. For this reason we make two comparisons; BEM vs. XBEM for $k=2$ in Fig. 17, and PUBEM vs. PUXBEM for $k=20$ in Fig. 18. We note that in all of the above examples, the reference solutions result from converged BEM schemes. Since it has been shown in Fig. 15 that XBEM and PUXBEM converge to the same result, with far fewer degrees of freedom; in this subsection we obtained reference solutions using converged XBEM and PUXBEM schemes respectively.

275 To produce the results in Fig. 17 and Fig. 18 we take $\gamma=0.1$ around a single corner, as shown in Fig. 16, in order to perform the evaluation close to a singularity where BEM and BEM are less effective. It is clear that we observe a similar increase in accuracy as seen in the previous results, in the region of two orders of magnitude. 


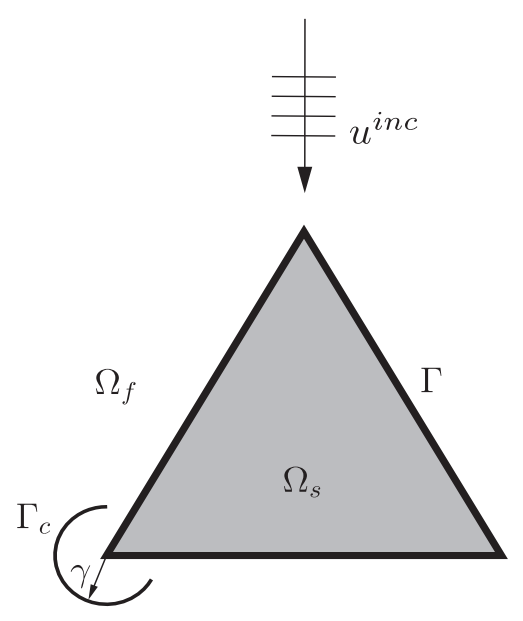

Figure 16. Internal point locations for single triangular scattering object.

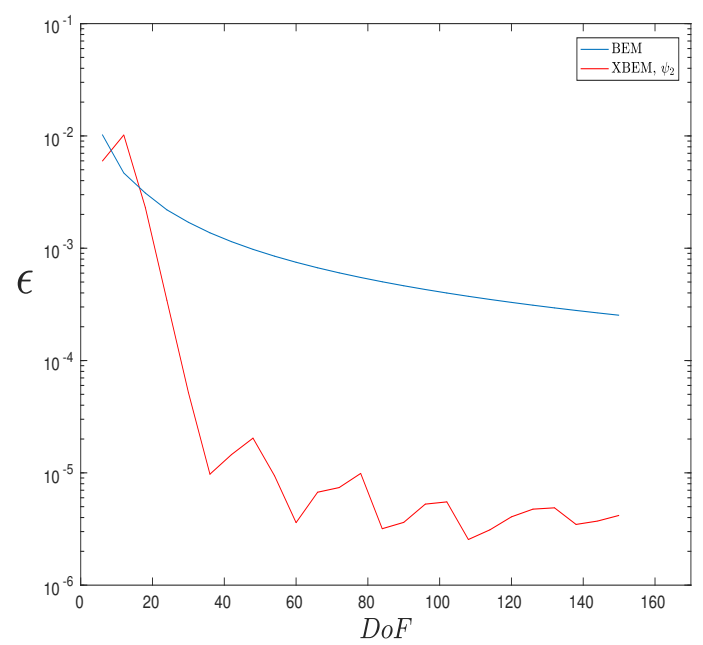

Figure 17. Relative error vs. DoF for $\gamma=0.1, k=2$.

\section{CONCLUSIONS}

Singular enrichment functions have been introduced for Helmholtz scattering by polygonal objects, and implemented in both XBEM and PUXBEM formulations for single and multiple scatterer cases. The enrichment functions are taken from the leading order term in the series of fractional-order Bessel functions in the asymptotic wave field close to a corner. The results provide evidence that the benefit of including singular basis functions for acoustic scattering from polygonal scattering objects can be extended successfully to BEM from other numerical methods. It is found preferable to introduce two enrichment degrees of freedom per corner, rather than one, to express the asymptotic field. Enrichment or the corners involves the addition of only this small number of degrees of freedom to the linear system, but offers approximately two orders of magnitude reduction in our error indicator.

The use of an approximation, $r_{b}^{\alpha}$, to the Bessel function markedly improves upon conventional $\mathrm{BEM}$, but fails to reach the accuracy available by using the actual Bessel function. It is clear that PUXBEM is consistently more accurate than XBEM for the set of problems tested, and also retains accuracy over a considerably larger frequency range than either BEM or XBEM for a fixed number of degrees of freedom. The use of blending functions to enforce continuity of potential is not found 


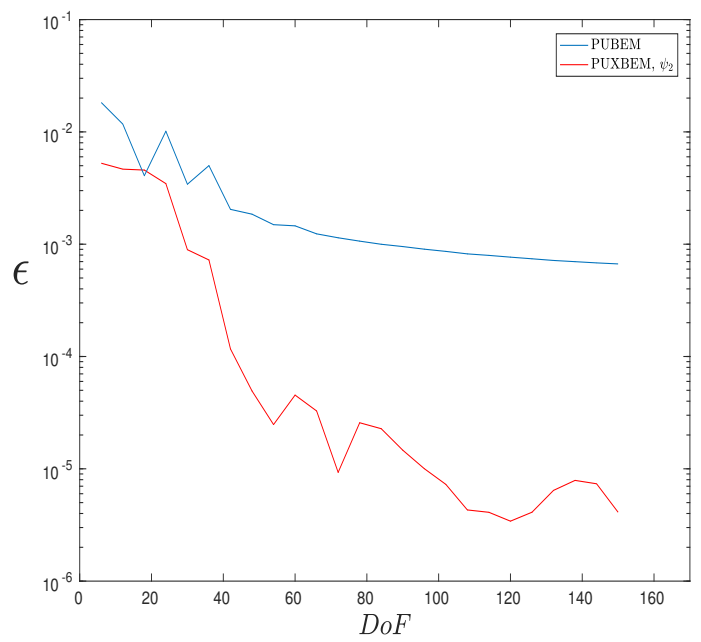

Figure 18. Relative error vs. DoF for $\gamma=0.1, k=20$.

to be helpful and the best results are achieved, at least for the medium to high frequency problems (his paper, without blending. This will introduce a small discontinuity in the solution but it is of negligible size because of the decay in the Bessel function over the length of the scatterer face. The conditioning of the BEM linear system deteriorates with the introduction of XBEM corner enrichment, but only moderately due to the small number of enrichment functions. The PUXBEM system is severely ill-conditioned, a feature inherited from the PUBEM use of the plane wave basis, but by use of a solver sympathetic to ill-conditioning the method gives the most accurate results of all.

One might speculate on the possibility of transferring the technique to $3 \mathrm{D}$ to consider scattering from polyhedral objects. The situation at the edges of such a scatterer resembles that in the $2 \mathrm{D}$ analysis of planar polygons in this paper, and we suggest that the Bessel function enrichment might be applied to edges to enhance accuracy in 3D. The situation at vertices of a polygon is not so clear. However, it may be that enriching the edges, and omitting any special treatment for vertices, might produce results of sufficient accuracy. This is an open subject for further research.

\section{REFERENCES}

1. Coifman, R., Rokhlin, V. and Wandzura, S., 1993. The fast multipole method for the wave equation: A pedestrian prescription. IEEE Antennas and Propagation Magazine, 35(3), pp.7-12.

2. Bebendorf, M., 2000. Approximation of boundary element matrices. Numerische Mathematik, 86(4), pp.565-589.

3. van't Wout, E., Glat, P., Betcke, T. and Arridge, S., 2015. A fast boundary element method for the scattering analysis of high-intensity focused ultrasound. The Journal of the Acoustical Society of America, 138(5), pp.2726-2737.

4. Khajah, T., Antoine, X. and Bordas, S., 2016. Isogeometric finite element analysis of time-harmonic exterior acoustic scattering problems. arXiv preprint arXiv:1610.01694.

5. El Kacimi, A., Laghrouche, O., Mohamed, M.S. and Trevelyan, J., 2018. Bernstein-Bzier based finite elements for efficient solution of short wave problems. Computer Methods in Applied Mechanics and Engineering.

6. Lieu, A., Gabard, G. and Briot, H., 2016. A comparison of high-order polynomial and wave-based methods for Helmholtz problems. Journal of Computational Physics, 321, pp.105-125.

7. Babuka, I. and Melenk, J.M., 1997. The Partition of Unity Method. International Journal for Numerical Methods in Engineering, 40(4), pp.727-758.

8. Laghrouche, O., Bettess, P. and Astley, R.J., 2002. Modelling of short wave diffraction problems using approximating systems of plane waves. International Journal for Numerical Methods in Engineering, 54(10), pp.1501-1533.

9. Laghrouche, O., Bettess, P., Perrey-Debain, E. and Trevelyan, J., 2005. Wave interpolation finite elements for Helmholtz problems with jumps in the wave speed. Computer Methods in Applied Mechanics and Engineering, 194(2-5), pp.367-381.

10. Farhat, C., Harari, I. and Franca, L.P., 2001. The discontinuous enrichment method. Computer Methods in Applied Mechanics and Engineering, 190(48), pp.6455-6479. 
11. Ladevze, P., Arnaud, L., Rouch, P. and Blanz, C., 2001. The variational theory of complex rays for the calculation of medium-frequency vibrations. Engineering Computations, 18(1/2), pp.193-214.

12. Cessenat, O. and Despres, B., 1998. Application of an ultra weak variational formulation of elliptic PDEs to the two-dimensional Helmholtz problem. SIAM Journal on Numerical Analysis, 35(1), pp.255-299.

13. Hiptmair, R., Moiola, A. and Perugia, I., 2011. Plane wave discontinuous Galerkin methods for the 2D Helmholtz equation: analysis of the p-version. SIAM Journal on Numerical Analysis, 49, pp.264-284.

14. Gabard, G., 2007. Discontinuous Galerkin methods with plane waves for time-harmonic problems. Journal of Computational Physics, 225, pp.1961-1984.

15. Colton, D. and Kress, R. 1993 , Integral Equation Methods in Scattering Theory, Springer.

16. Perrey-Debain, E., Trevelyan, J. and Bettess, P., 2004, 2002. New special wave boundary elements for short wave problems. Communications in Numerical Methods in Engineering, 18(4), pp.259-268.

17. Perrey-Debain, E., Trevelyan, J. and Bettess, P., 2004. Wave boundary elements:a theoretical overview presenting applications in scattering of short waves, Eng. Anal Bound. Elem., 28: 131-141.

18. Perrey-Debain, E., Laghrouche, O., Bettess, P. and Trevelyan, J., 2004. Plane-wave basis finite elements and boundary elements for three-dimensional wave scattering. Philosophical Transactions of the Royal Society of London A: Mathematical, Physical and Engineering Sciences, 362(1816), pp.561-577.

19. Chandler-Wilde, S.N. and Langdon, S., 2007. A Galerkin boundary element method for high frequency scattering by convex polygons. SIAM Journal on Numerical Analysis, 45(2), pp.610-640.

20. Groth, S.P., Hewett, D.P. and Langdon, S., 2015. Hybrid numericalasymptotic approximation for high-frequency scattering by penetrable convex polygons. IMA Journal of Applied Mathematics, 80(2), pp.324-353.

21. Helsing, J. and Ojala, R., 2008. Corner singularities for elliptic problems: Integral equations, graded meshes, quadrature, and compressed inverse preconditioning. Journal of Computational Physics, 227(20), pp.8820-8840.

22. migaj, W., Betcke, T., Arridge, S., Phillips, J. and Schweiger, M., 2015. Solving boundary integral problems with BEM++. ACM Transactions on Mathematical Software (TOMS), 41(2), p.6.

23. Platte, R.B. and Driscoll, T.A., 2004. Computing eigenmodes of elliptic operators using radial basis functions. Computers Mathematics and Applications, 48(3-4), pp.561-576.

24. Luostari, T., Huttunen, T. and Monk, P., 2012. The ultra weak variational formulation using Bessel basis functions. Communications in Computational Physics, 11(2), pp.400-414.

25. Barnett, A.H. and Betcke, T., 2010. An exponentially convergent nonpolynomial finite element method for timeharmonic scattering from polygons. SIAM Journal on Scientific Computing, 32(3), pp.1417-1441.

26. Antunes, P.R.S. and Valtchev, S.S., 2010. A meshfree numerical method for acoustic wave propagation problems in planar domains with corners and cracks. Journal of Computational and Applied Mathematics, 234(9), pp.26462662.

27. Chaumont-Frelet, T. and Nicaise, S., 2018. High-frequency behaviour of corner singularities in Helmholtz problems. ESAIM: Mathematical Modelling and Numerical Analysis, 52(5), pp.1803-1845.

28. A. A. Becker, 1992. The boundary element method in engineering: a complete course, McGraw-Hill, Maidenhead.

29. Schenck, H.A., 1968. Improved integral formulation for acoustic radiation problems. The journal of the acoustical society of America, 44(1), pp.41-58.

30. Burton, A.J. and Miller, G.F., 1971. The application of integral equation methods to the numerical solution of some exterior boundary-value problems. Proc. R. Soc. Lond. A, 323(1553), pp.201-210.

31. Li, S. and Huang, Q., 2010. An improved form of the hypersingular boundary integral equation for exterior acoustic problems. Engineering Analysis with Boundary Elements, 34(3), pp.189-195.

32. Liu, Y. and Rizzo, F.J., 1997. Scattering of elastic waves from thin shapes in three dimensions using the composite boundary integral equation formulation. The Journal of the Acoustical Society of America, 102(2), pp.926-932.

33. Descloux, J. and Tolley, M., 1983. An accurate algorithm for computing the eigenvalues of a polygonal membrane. Computer Methods in Applied Mechanics and Engineering, 39(1), pp.37-53.

34. Antunes, P.R.S., 2018. A numerical algorithm to reduce the ill conditioning in meshless methods for the Helmholtz equation. Numerical Algorithms, 79(3), pp.879-897. 